\title{
Radio-optical Identification of Very-Steep Spectrum Radio Sources from the UTR-2 Catalogue
}

\author{
H. Andernach \\ Depto. de Astronomía, Apdo. Postal 144, Univ. Guanajuato, \\ Guanajuato, Mexico \\ O.V. Verkhodanov and N.V. Verkhodanova \\ Special Astrophysical Observatory, Nizhnij Arkhyz, Russia, 369167
}

\begin{abstract}
We used radio source catalogues accessible from the CATS database to establish radio continuum spectra for decametric radio sources in the UTR-2 catalogue. In an attempt to find further candidates for high-redshift radio galaxies, we searched the FIRST and NVSS surveys for counterparts of a sample of UTR sources with ultra-steep radio spectra (USS, $\alpha \leq-1.2, \mathrm{~S} \sim \nu^{\alpha}$ ). We derived accurate positions and sizes for 23 of these USS sources. The search for optical counterparts from the APM (object) and DSS (image) databases, as well as infrared and X-ray identifications of these UTR sources are in progress.
\end{abstract}

A radio survey obtained with the UTR telescope (Kharkov, Ukraine) at frequencies $10-25 \mathrm{MHz}$ has resulted in a catalogue of 1822 sources (Braude et al. 1978-1994; www.ira.kharkov.ua/UTR2). Covering about $30 \%$ of the sky north of $-13^{\circ}$ declination, this survey is presently the lowest-frequency source catalogue of its size, and provides an ideal basis to improve the poor optical identification content of sources selected at decametric frequencies. In a separate paper (Verkhodanov et al., these proceedings) we described our goal to identify all UTR sources with known radio sources and to search for optical counterparts on the Digitzed Sky Surveys. To this aim we used the CATS database (http://cats.sao.ru, Verkhodanov et al. 1997a) and derived for each UTR source between one and four radio counterparts which contribute significantly to the UTR source flux.

We were able to fit spectra for all but 7 of the 2314 radio counterparts to the 1822 UTR sources. For $39 \%$ of these counterparts the best fit was a straight (S) spectrum, for $8 \%$ it was a convex $\left(\mathrm{C}^{-}\right)$curve, and for $53 \%$ it was a concave $\left(\mathrm{C}^{+}\right)$ curve in the $\log \nu-\log \mathrm{S}$ plot. Here we present our first results on a subsample of ultra-steep spectrum (USS) sources (spectral index $\alpha \leq-1.2, \mathrm{~S} \sim \nu^{\alpha}$ ). This class of sources is being actively studied by various groups (Parijskij et al. 1996; Röttgering et al. 1997; McCarthy et al. 1997), mainly because they are often identified with very distant radio galaxies, which are probes of the early Universe and thought to be indicators of proto-clusters (e.g. Djorgovski 1987).

In our catalogue of 2314 radio counterparts (the full list will be published elsewhere) there are 422 S-type sources with "very-steep spectrum" (VSS, $\alpha \leq$ -1.0 ), and for the present work we selected from these a subsample of 102 
"ultra-steep spectrum" (USS) objects $(\alpha \leq-1.2)$. To further increase the radiopositional accuracy for a subsequent optical identication, we searched for radio counterparts of USS sources in the February 1998 version of the FIRST catalogue (http://sundog.stsci.edu/first/catalogs). This yielded 38 FIRST counterparts for 23 USS sources in UTR.

To check the overall structure of these FIRST counterparts we also extracted source parameters for these from the lower-resolution NVSS survey at $1.4 \mathrm{GHz}$ (http://www.cv.nrao.edu/NVSS/). The deconvolved angular size (LAS) of these counterparts in NVSS range from "unresolved" $\left(<19^{\prime \prime}\right)$ up to 83 ". Twelve of the 23 UTR USS sources have single counterparts in FIRST and NVSS, with deconvolved sizes between $2^{\prime \prime}$ and $9^{\prime \prime}$ in the FIRST catalogue.

The more extended sources in NVSS tend to break up into several components in the FIRST catalogue, e.g. into three (GR 0858-02), four (GR 0135-08, GR 1731+43) or even six components (GR 0257-08). As expected, for the larger source complexes we usually find a larger NVSS/FIRST flux ratio, most likely due to missing flux in the FIRST survey. Interesting enough, all except one of the 38 FIRST components are resolved by the FIRST beam of $\sim 5^{\prime \prime}$, i.e. have a deconvolved major axis of $>3^{\prime \prime}$.

For some of the sources an optical identification has been made previously. The NASA Extragalactic Database (NED, http://nedwww.ipac.caltech.edu) suggests that the complex source GR 0135-08 may be identified with the $z=0.041$ galaxy MCG-02-05-020, while GR 1214-03 coincides with a QSO at $\mathrm{z}=0.184$ from the Las Campanas Redshift Survey (LCRS) and a radio size of only 3", and GR $1243+04$ is a radio galaxy $(4 \mathrm{C}+03.24)$ at $\mathrm{z}=3.57$. The FIRST maps of GR $0910+48$ and GR $0942+54$ show them to be radio doubles of LAS $=30^{\prime \prime}$ and $10^{\prime \prime}$, respectively. Images from the second-epoch Digitized Sky Survey (DSS-2, http://archive.stsci.edu/dss/dss_form.html) do not reveal any optical counterpart above the plate limit near the symmetry centre of these sources, thus they appear to be good candidates for distant radio galaxies. For the optical identification of the other sources we are employing available resources like object catalogues from APM (http://www.ast.cam.ac.uk/ apmcat) and COSMOS (Drinkwater et al. 1995) as well as the image archive of DSS-2.

\section{References}

Braude S.Ya. et al. 1978, Ap\&SS, 54, 37; 1979, Ap\&SS, 64, 73; 1981, Ap\&SS, 74, 409; 1985, Ap\&SS, 111, 1; 1994, Ap\&SS, 213, 1.

Djorgovski S., 1987, in Nearly Normal Galaxies, ed. S. Faber (Springer-Verlag), p. 290

Drinkwater M.J. et al. 1995, PASA 12, 248

McCarthy P.J. et al. 1997, ApJS, 112, 415

Parijskij Yu.N. et al. 1996, Bull. SAO, 40, 5

Röttgering H.J.A. et al. 1997, A\&A 326, 505

Verkhodanov O.V. et al. 1997, ASP Conf. Ser. 125, 322, eds. G. Hunt \& H.E. Payne, ASP, San Francisco. 